\title{
Nanocurcumin potential to increase mitochondrial biogenesis in skeletal muscle via AMPK-PGC-1alpha
}

DOI:10.36909/jer.ASSEEE.16073

\author{
Hamidie Ronald D. Ray ${ }^{1}$, Asep Bayu Dani Nandiyanto ${ }^{2}$, Rita Patriasih ${ }^{3}$, Abdullah Firmansah ${ }^{4}$ \\ ${ }^{1}$ Faculty of Sport and Health Education, Universitas Pendidikan Indonesia, Bandung, \\ Indonesia \\ ${ }^{2}$ Departemen Kimia, Universitas Pendidikan Indonesia, Bandung, Indonesia \\ ${ }^{3}$ Faculty of Technology and Vocational Education, Universitas Pendidikan Indonesia, \\ Bandung, Indonesia \\ ${ }^{4}$ Faculty of Medicine, Universitas Padjadjaran, Jatinangor, Indonesia
}

*Corresponding Author: hamidieronald@upi.edu

\begin{abstract}
Curcumin, which comes from the rhizome Curcuma longa L, is known as the polyphenol with a high level content of natural antioxidants and has benefits on pharmacological activities and human health. The purpose of current study was to investigate the effect of nanocurcumin itself and the combination with exercise on 5' adenosine monophosphate-activated protein kinase (AMPK), PGC-1 $\alpha$, and mitochondrial protein expression of cytochrome c oxidase subunit IV (COX-IV), in rat gastrocnemius muscle. Animals separated to be non-endurance exercise and endurance exercise group. Curcumin and nanocurcumin with doses $100 \mathrm{mg}$ per kg-body weight per day were given peroral in both groups for 28 days in order to determine the effect of nanocurcumin on mitochondrial markers including AMPK-PGC-1 $\alpha$ and COXIV. Western blotting (WB) method was applied to investigate the protein expression on skeletal muscle. The result showed that nanocurcumin increased mitochondrial marker protein
\end{abstract}


COX-IV on non exercise and exercise groups. Furthermore, our result demonstrated that nanocurcumin treatment combined with exercise increased the phosphorylation of AMPK and PGC-1 $\alpha$. Addition, nanocurcumin treatment alone, without exercise, also increased PGC-1 $\alpha$ protein expression. This current result suggests that nanocurcumin could increase mitochondrial biogenesis markers. When it is used together with exercise, it potentially has the additive effect of exercise to increase mitochondrial markers through AMPK-PGC-1 $\alpha$ signaling pathway. In conclusion, nanocurcumin treatment combined with exercise potentially increases mitochondrial biogenesis.

Key words: endurance training; mitochondria; nanocurcumin; skeletal muscle. 


\section{INTRODUCTION}

One of the largest part of human body is skeletal muscle. Skeletal muscle has a huge potential in responding to stress physiology in our body. One example of adaptation of skeletal muscle is in the event of mitochondrial biogenesis that occurs during exercise. The purpose of mitochondrial biogenesis is to clean up damaged mitochondria so that it will improve health. This will prevent the imbalance of metabolism, which if this imbalance occurs, it will cause the onset of diseases, such as overweight, diabetes mellitus, heart failure and blood vessel diseases, and premature aging. Exercise activities are likely to increase the characteristics of metabolism in the skeletal muscle. It can also occur in increasing of quantity and quality of mitochondria and the transport of expression proteins from glucose transporter 4 (GLUT4) expression (Holloszy, 2008). Peroxisome proliferator-activated receptor gamma coactivator 1 (PGC-1 $\alpha$ mitochondrial marker protein COX-IV in the gastrocnemius) is known as the main regulator in mitochondrial biogenesis. Furthermore, PGC-1 $\alpha$ have ability to work with nuclear respiratory factor 2 (NRF-2/GA-binding proteinA) and nuclear respiratory factor 1 (NRF1) to increasing transcription factor A, mitochondrial (Tfam). This condition will regulated mitochondria DNA transcription, translation, and repair through transcription in mitochondrial genes (Hock \& Kralli., 2009). Previous study indicated that exercise ability increased the PGC- $1 \alpha$ mRNA and protein expression levels (Terada et al., 2004; Taylor et al., 2005; Russell et al., 2003). This condition speculated that exercise needs PGC- $1 \alpha$ to be a regulator of adaptation on metabolic activity.

NAD-dependent deacetylase sirtuin-1(SIRT-1) is a member of the NAD+ dependent deacetylates family and ADP-ribosyltransferase that is found to be involved in several fundamental processes within the cell, including metabolism regulation and DNA repair (Baur et al., 2010; Donmez \& Guarente, 2010). SIRT-1 will be involved in mitochondrial 
biogenesis process via decreasing acetylation of PGC-1 $\alpha$. On the other side, AMP-activated protein kinase (AMPK), one of metabolism sensor has the ability to activate PGC-1 expression (Suwa et al., 2008). Similar to AMPK, SIRT-1 also has a broad effect on energy metabolism and has implications for the functioning of mitochondria and the activeness of several enzymes that have an effect on increasing mitochondrial capacity of biogenesis and performance. The above statements give the understanding of the adaptation results of metabolism as well as the result of exercise activities through increasing of PGC-1 $\alpha$ protein expression via AMPK-SIR-1 pathways. Furthermore, the NAD+-dependent histone deacetylase SIRT-1 is increased by exercise especially acute training conditions. (Lagouge et al., 2006).

Some types of polyphenols are rumored to also activate SIRT-1 in Vitro. They further state that the substance has the potential to increase mitochondrial biogenesis through deacetylation of PGC-1 $\alpha$ (Davis et al., 2009). These polyphenols are components of Curcuma longa L. C. longa is also the source of curcumin, has medical advantages, and is very popular in Asia. Previous research has shown that curcumin has higher antioxidant properties than resveratrol (Eckert et al., 2013). One experiment was used mouse prone 8 (SAMP8) for long-term administration of curcumin in everyday diet succeed to delay fast aging through increasing mitochondrial biogenesis in the brain included mitochondrial membrane, ATP levels and mitochondrial fusion (Cotman et al., 2002). However, the benefits and how work of nanocurcumin to increasing mitochondrial have never been investigated.

In our current experiment we are interested to investigate the mechanism of nanocurcumin in improving mitochondrial biogenesis. We hypothesize that nanocurcumin has a good effect and increases mitochondrial biogenesis skeletal muscle and, when it is combined with exercise, nanocurcumin synergistically increases the effects of exercise in improving 
mitochondrial biogenesis in gastrocnemius muscles.

\section{METHOD}

The type of experimental design carried out was completely randomized design. The procedure followed the Ethics Committee on Animal Experimentation of Universitas Kristen Maranatha (No: 098/KEP/VII/2020). Adult Wistar rats aged 10 weeks were used in this experiment. Animals were placed in rom with consistency temperature by air-conditioned and used the lamp by setting 12 hours bright and 12 hours dark. Diet given was ad libitum. Furthermore, rats were randomly divided into 6, including groups of non-exercise and exercise groups. Each group was further divided into control groups, one group got curcumin (100 milligram/kg-body weight/day) and one group got nanocurcumin (100 milligram/kgbody weight/day). All animals got treatment through oral pathways for 24 days, while the control got fluid equal to treatment (vehicle alone). The type of endurance exercise given was swimming exercise for 2 hours divided into 4 sets. The duration of each set was 30 minutes with a 5-minute break at each set. After the first 30 minutes, loading of $2 \%$ from the body weight was used to the animals by wrapping the body with a plastic cable. The swimming pool was $50 \mathrm{~cm}$ deep and the swimming area was $200 \mathrm{~cm} 2 /$ rat. Animals exercised for twentyfour days, six times a week. After completion of the treatment period, animals were given 50 $\mathrm{mg}$ of Pentobarbital per every $100 \mathrm{mg}$ of body weight. After that, for the examination of protein expression, gastrocnemius muscle was quickly taken and isolated. The tissue was washed and cleaned from other organ which not needed and immediately put in $\mathrm{NaCl}$ solution (Cotman et al., 2007). Generally $40 \mathrm{mg}$ gastrocnemius muscle was homogenized in Buffer A which made from $250 \mathrm{mM}$ sucrose, $10 \mathrm{mM} \mathrm{NaCl}, 3 \mathrm{mM} \mathrm{MgCl} 2,1 \mathrm{mmol} / \mathrm{l}$ dithiothreitol (DTT) and place on $500 \mu \mathrm{l}$ of ice-cold for 30 second, after that $1 \mathrm{mM}$ phenylmethylsulphonyl fluoride (PMSF), and $2 \mu 1$ protease inhibitor cocktail added on Buffer A. Centrifuged used at $500 \mathrm{~g}$ at $4^{\circ} \mathrm{C}$ in order to get supernatant on the homogenized procedure. This conditions knows as the whole fraction. Furthermore, The remaining pellet was resuspended in $500 \mu \mathrm{l}$ of ice-cold buffer B which contains of $50 \mathrm{mM}$ Tris, pH 7.5, 1 mM EDTA, $1 \mathrm{mM}$ EGTA, $1 \mathrm{mM}$ DTT, $50 \mathrm{mM} \mathrm{NaF}, 5 \mathrm{mM}$ Na pyrophosphate, $50 \mathrm{mM} \mathrm{MgCl} 2,10 \%$ glycerol, $1 \%$ Triton $\mathrm{X}-100$, $1 \mathrm{mM}$ PMSF, and after that $2 \mu \mathrm{l}$ tissue protease inhibitor cocktail added on that buffer). The buffer incubated with mixing for every 1 minutes on cold condition for 10 minutes. The 
resuspended pellet which get from this protocol take on centrifuge and spin at $3100 \mathrm{~g}$ at $4^{\circ} \mathrm{C}$ for 5 or 6 minutes..

Analysis used western blotting following the previously described process (Nybo et al., 2003). Briefly, a number of proteins from the sample were included in the SDS-Page gel with a concentration for AMPK- $\alpha$ (Cell Signaling Technology, Danvers, MA, USA), PGC$1 \alpha$ (Calbiochem, San Diego, CA, USA), COX-IV (Abcam, Cambridge, England), GAPDH (Abcam, Cambridge, England). Furthermore, protein through the PVDF membrane was carried out into the transfer process. Membrane was incubated in a blocking buffer and given primary antibodies at $4^{\circ} \mathrm{C}$ overnight. The membranes were then reacted with a secondary antibody. The next step was the membrane reacted with a secondary antibody. Furthermore, the analysis protein signal used by Amersham ECL western blotting detection reagent chemiluminescence (GE Healthcare, Piscataway, NJ, USA). In order to quantified inetensity we used imaging software (Image J, version 1.46, NIH, Maryland, USA).

\section{RESULTS AND DISCUSSION}

\section{Results}

Values are described as the mean $\pm \mathrm{SD}, \mathrm{n}=6$. *: significant difference than control group $(\mathrm{p}<$ 0.05) \#: significant difference than control + exercise group $(\mathrm{p}<0.05)$. Cur $=$ curcumin 100 milligram $/ \mathrm{kg} /$ body-weight/day, NCur $100=$ nanocurcumin 100 milligram $/ \mathrm{kg} / \mathrm{body}-$ weight/day. These results indicate that nanocurcumin treatment combined with endurance training increased AMPK expression in the gastrocnemius. 

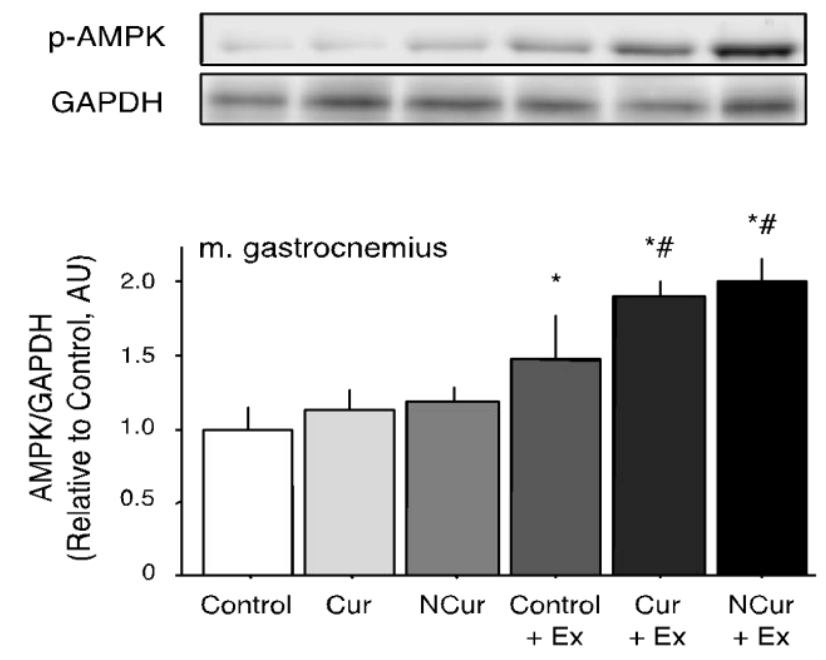

Figure 2 Immunoblots for markers of AMPK Expression.

Values are described as the mean $\pm \mathrm{SD}, \mathrm{n}=6$. *: significant difference than control group ( $\mathrm{p}$ $<0.05)$ \#: significant difference than control + exercise group $(\mathrm{p}<0.05)$. Cur $=$ curcumin 100 milligram/kg/body-weight/day, NCur $100=$ nanocurcumin 100 milligram $/ \mathrm{kg} / \mathrm{body}-$ weight/day. These results indicate that nanocurcumin treatment combined with endurance training increased expression of PGC-1 $\alpha$ in the gastrocnemius muscles.

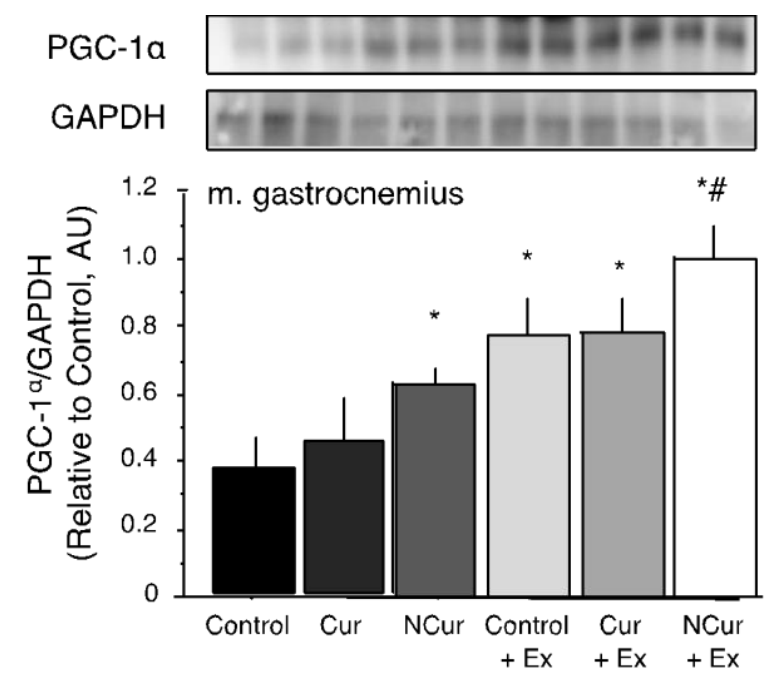

Figure 2 Expression of PGC- $1 \alpha$.

Values are described as the mean $\pm \mathrm{SD}, \mathrm{n}=6$. *: significant difference than control group $(\mathrm{p}$ $<0.05)$ \#: significant difference than control + exercise group $(\mathrm{p}<0.05)$. Cur $=$ curcumin 100 
milligram/kg/body-weight/day, NCur 100 = nanocurcumin $100 \mathrm{mg} / \mathrm{kg}-\mathrm{BW} /$ day. These results indicate that nanocurcumin treatment combined with endurance training increased the expression of the mitochondrial marker (COX-IV).

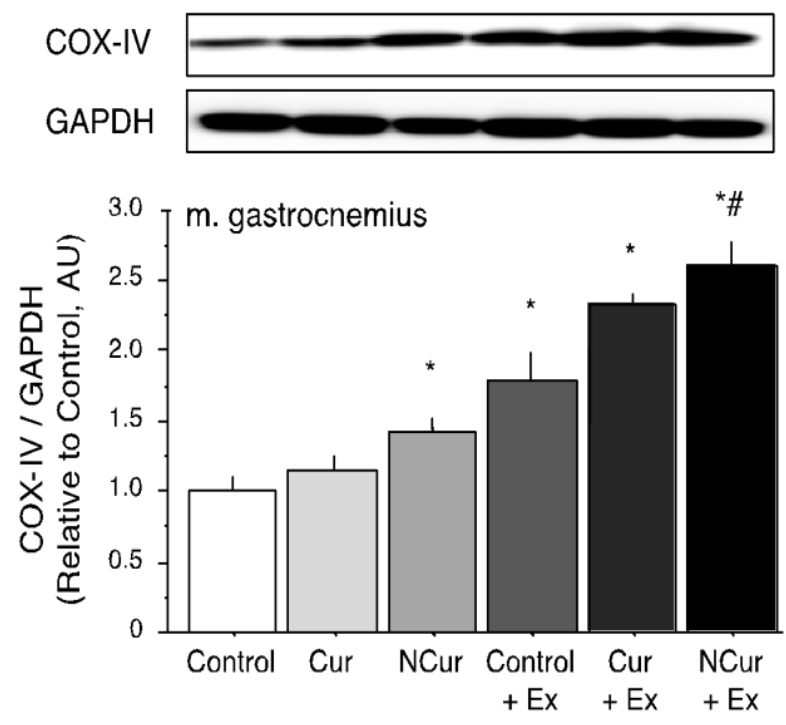

Figure 3 Immunoblots for a mitochondria marker (COX-IV).

\section{Discussions}

In this current investigation, we were interested to determine the benefit of nanocurcumin treatment itself and the combined effect of curcumin and exercise to identify their impact on mitochondrial biogenesis in skeletal muscles. Since there is limited study on curcumin effect to regulate mitochondrial biogenesis in skeletal muscles, the goal of the present investigation was to determine whether both nanocurcumin and exercise could up-regulate mitochondrial biogenesis. Since, we know that this is the first study to provide evidence of mitochondrial biogenesis in skeletal muscles treated with curcumin following endurance training in rats. Our hypothesis is that nanocurcumin components have the ability to regulate mitochondrial biogenesis was supported by the increase in COX-IV expression in Gas (Fig. 3). Furthermore, it is interesting that nanocurcumin treatment augmented the endurance training induced induction of mitochondrial markers (Fig. 1). Previous research had suggested that polyphenols 
could regulated mitochondria biogenesis through their antioxidant activity. Therefore, we suggested that active substrate of nanocurcumin in muscles would be exerted through the regulation of increasing of mitochondria (mitochondrial biogenesis). In order to obtain insights into the mechanisms of curcumin-stimulated mitochondrial biogenesis, we investigated PGC-1 $\alpha$ protein expression in response to nanocurcumin administration and endurance exercise.

PGC-1 $\alpha$ coactivator regulated to activating the nuclear and transcription of mitochondria to enhance mitochondrial biogenesis and regulation of genes which involved lipid metabolism and oxidative phosphorylation (Benton et al., 2008). On the other way, PGC-1 $\alpha$ can also coactivate several types of transcriptional factors including NRF-1 and Tfam (Lin et al., 2002). In previous research conducted on animals with PGC- $1 \alpha \mathrm{KO}$, it clearly or significantly reduced expression of Tfam and COX-IV (Wu et al., 1999). Therefore, we investigated differences in the PGC-1 $\alpha$ in gastrocnemius muscle between groups that received curcumin treatment or nanocurcumin itself and combination with exercise. As well as in our previous research. Current research also shows that nanocurcumin increased PGC-1 $\alpha$. Interestingly, when it was combined with exercise, it turns out that nanocurcumin significantly increased PGC-1 $\alpha$ expression (Fig 2). Based on these data, nanocurcumin treatment potentially gives high impact in mitochondrial biogenesis through PGC-1 $\alpha$ when metabolic demands involve skeletal muscle on endurance exercise. Furthermore, nanocurcumin may have the ability to exert an additive or synergistic effect with endurance exercise.

In skeletal muscles, SIRT-1 activated PGC-1 $\alpha$ deacetylation, which was the essential point to increase mitochondrial biogenesis. Interestingly, several polyphenols, including resveratrol and curcumin, have an ability to activate SIRT1 in a variety of experiments, directly or indirectly (Price et al., 2012). Another study suggests that curcumin regulated myocardial IRinduces mitochondrial oxidative damage through increasing SIRT-1 protein expression 
(Queen \& Tollefsbol, 2010). This provides an opportunity to further probing the effects of polyphenols on targets through a SIRT-1-PGC-1 complex or other pathway in order to regulate mitochondrial biogenesis on skeletal muscle that resembles the effects of endurance exercise.

As the statement above, the activation of PGC-1 by SIRT-1 is an important step although the mechanism of how the expression SIRT-1 improves in endurance exercise is still not clearly explained. Another way is AMPK, since its effect increases by endurance exercise (Aziz, 2012). In addition, our research shows that the administration of nanocurcumin increased AMPK expression, while combination of endurance exercise and nanocurcumin significantly increased AMPK expression (Fig. 1). It may be able to explain synergistically the effects of combination treatment in vivo. This discovery demonstrates that the administration of nanocurcumin has a better effect than just the administration of curcumin and potential to induce the function of mitochondrial biogenesis which may be dependent on the presence of SIRT-1. The previous study of resveratrol also showed similar results which involved SIRT-1 and AMPK (Boily et al., 2009). This provides clarity on the dynamic interaction between the two pathways, AMPK is seen to activate SIRT-1 and possibly through indirect improvement in NAD+ cell concentration) (Boily et al., 2008).

Endurance exercise increases mitochondrial biogenesis and potential in improving performance by increasing oxidative capacity (Canto et al., 2009). AMPK enzyme serves as a metabolic sensor. Structurally, AMPK is the heterotrimer of three subunits: one catalytic $(\alpha)$ and two regulatory $(\beta$ and $\gamma$ ) subunits. Exercise increases [AMP] / [ATP] ratio in cells and activates AMPK (Cheung et al., 2003). Further administration of curcumin showed the increase of AMPK phosphorylation and improved insulin resistance (Hardie et al., 2001). Curcumin also increased AMPK phosphorylation in research on adipocyte with mice by administering a high-fat diet (Na et al., 2011); It seems that AMPK is the main target in 
curcumin effect to increase mitochondrial biogenesis. Since the energy state progresses due to endurance exercise, connection of AMPK and SIRT1 through augmented in the cellular $\mathrm{NAD}+$ level can change the expression of proteins in order to increase mitochondria biogenesis.

Based on the current results, we speculated that endurance exercise will activate AMPK which following by regulated SIRT1. Furthermore, AMPK will be increasing protein expression PGC-1 $\alpha$. Finally, this effect will regulate mitochondrial biogenesis, including COX-IV protein expression (Fig.3). Addition of nanocurcumin seems to have a similar effect with endurance exercise to increase mitochondrial biogenesis through AMPK and PGC-1 $\alpha$. Further investigation is necessary to clarify the potential for an additive or synergistic effect of curcumin treatment and endurance exercise over nanocurcumin treatment alone, including resolution of the possibility that AMPK phosphorylation is increased by curcumin-mediated augmentation of LKB-1 phosphorylation up-stream of the effect of AMPK regulation on skeletal muscles (Ejaz et al., 2009).

\section{CONCLUSION}

This current study indicated that nanocurcumin together with endurance exercise increase AMPK. Activation of AMPK regulated PGC-1 $\alpha$ protein expression and involve on increasing COX-IV protein expression. This data suggests that nanocurcumin has a possibility to enhance performance through AMPK and PGC-1 $\alpha$ pathway.

\section{ACKNOWLEDGEMENTS}

This research was supported by Lembaga Pengelola Dana Pendidikan (LPDP) Indonesia through RISPRO INVITASI SCHEME. 


\section{REFERENCES}

Aziz, M. T. A., Motawi, T., Rezq, A., Mostafa, T., Fouad, H. H., Ahmed, H. H., \& ElShafiey, R. 2012. Effects of a water-soluble curcumin protein conjugate vs. pure curcumin in a diabetic model of erectile dysfunction. The journal of sexual medicine, 9(7): 18151833.

Benton, C. R., Nickerson, J. G., Lally, J., Han, X. X., Holloway, G. P., Glatz, J. F., \& Bonen, A. 2008. Modest PGC-1 $\alpha$ overexpression in muscle in vivo is sufficient to increase insulin sensitivity and palmitate oxidation in subsarcolemmal, not intermyofibrillar, mitochondria. Journal of Biological Chemistry, 283(7): 4228-4240.

Boily, G., He, X. H., Pearce, B., Jardine, K., \& McBurney, M. W. 2009. SirT1-null mice develop tumors at normal rates but are poorly protected by resveratrol. Oncogene, 28(32), 2882-2893.

Boily, G., Seifert, E. L., Bevilacqua, L., He, X. H., Sabourin, G., Estey, C., \& McBurney, M. W. 2008. SirT1 regulates energy metabolism and response to caloric restriction in mice. PloS one, 3(3): e1759.

Cantó, C., Gerhart-Hines, Z., Feige, J. N., Lagouge, M., Noriega, L., Milne, J. C., \& Auwerx, J. 2009. AMPK regulates energy expenditure by modulating NAD+ metabolism and SIRT1 activity. Nature, 458(7241): 1056-1060.

Cheung, P. C., Salt, I. P., Davies, S. P., Hardie, D. G., \& Carling, D. 2000. Characterization of AMP-activated protein kinase $\gamma$-subunit isoforms and their role in AMP binding. Biochemical Journal, 346(3): 659-669. 
Cotman, C. W., \& Berchtold, N. C. 2002. Exercise: a behavioral intervention to enhance brain health and plasticity. Trends in Neurosciences, 25(6): 295-301.

Cotman, C. W., Berchtold, N. C., \& Christie, L. A. 2007. Exercise builds brain health: key roles of growth factor cascades and inflammation. Trends in Neurosciences, 30(9): 464472.

Davis, J. M., Murphy, E. A., Carmichael, M. D., \& Davis, B. 2009. Quercetin increases brain and muscle mitochondrial biogenesis and exercise tolerance. American Journal of Physiology-Regulatory, Integrative and Comparative Physiology, 296(4): R1071-R1077.

Donmez, G., \& Guarente, L. 2010. Aging and disease: connections to sirtuins. Aging cell, 9(2): 285-290.

Eckert, G. P., Schiborr, C., Hagl, S., Abdel-Kader, R., Müller, W. E., Rimbach, G., \& Frank, J. 2013. Curcumin prevents mitochondrial dysfunction in the brain of the senescence-accelerated mouse-prone 8. Neurochemistry International, 62(5): 595-602.

Ejaz, A., Wu, D., Kwan, P., \& Meydani, M. 2009. Curcumin inhibits adipogenesis in 3T3L1 adipocytes and angiogenesis and obesity in C57/BL mice. The Journal of nutrition, 139(5): 919-925.

Hardie, D. G., \& Hawley, S. A. 2001. AMP-activated protein kinase: the energy charge hypothesis revisited. Bioessays, 23(12): 1112-1119.

Hock, M. B., \& Kralli, A. 2009. Transcriptional control of mitochondrial biogenesis and function. Annual review of physiology, 71: 177-203.

Holloszy, J. O. 2008. Regulation by exercise of skeletal muscle content of mitochondria and GLUT4. J Physiol Pharmacol, 59(Suppl 7): 5-18. 
Hood, D. A. 2001. Invited Review: contractile activity-induced mitochondrial biogenesis in skeletal muscle. Journal of Applied Physiology, 90(3): 1137-1157.

Lagouge, M., Argmann, C., Gerhart-Hines, Z., Meziane, H., Lerin, C., Daussin, F., \& Auwerx, J. 2006. Resveratrol improves mitochondrial function and protects against metabolic disease by activating SIRT1 and PGC-1 $\alpha$. Cell, 127(6): 1109-1122.

Lin, J., Wu, H., Tarr, P. T., Zhang, C. Y., Wu, Z., Boss, O., ... \& Spiegelman, B. M. 2002. Transcriptional co-activator PGC-1 $\alpha$ drives the formation of slow-twitch muscle fibres. Nature, 418(6899): 797-801.

Na, L. X., Zhang, Y. L., Li, Y., Liu, L. Y., Li, R., Kong, T., \& Sun, C. H. 2011. Curcumin improves insulin resistance in skeletal muscle of rats. Nutrition, Metabolism and Cardiovascular Diseases, 21(7): 526-533.

Nybo, L., Møller, K., Pedersen, B. K., Nielsen, B., \& Secher, N. H. 2003. Association between fatigue and failure to preserve cerebral energy turnover during prolonged exercise. Acta physiologica scandinavica, 179(1): 67-74.

Price, N. L., Gomes, A. P., Ling, A. J., Duarte, F. V., Martin-Montalvo, A., North, B. J., \& Sinclair, D. A. 2012. SIRT1 is required for AMPK activation and the beneficial effects of resveratrol on mitochondrial function. Cell metabolism, 15(5): 675-690.

Queen, B. L., \& Tollefsbol, T. O. 2010. Polyphenols and aging. Current Aging Science, 3(1): $34-42$.

Russell, A. P., Feilchenfeldt, J., Schreiber, S., Praz, M., Crettenand, A., Gobelet, C., \& Dériaz, O. 2003. Endurance training in humans leads to fiber type-specific increases in 
levels of peroxisome proliferator-activated receptor- $\gamma$ coactivator- 1 and peroxisome proliferator-activated receptor- $\alpha$ in skeletal muscle. Diabetes, 52(12): 2874-2881.

Suwa, M., Nakano, H., Radak, Z., \& Kumagai, S. 2008. Endurance exercise increases the SIRT1 and peroxisome proliferator-activated receptor $\gamma$ coactivator- $1 \alpha$ protein expressions in rat skeletal muscle. Metabolism, 57(7): 986-998.

Taylor, E. B., Lamb, J. D., Hurst, R. W., Chesser, D. G., Ellingson, W. J., Greenwood, L. J., \& Winder, W. W. 2005. Endurance training increases skeletal muscle LKB1 and PGC$1 \alpha$ protein abundance: effects of time and intensity. American Journal of PhysiologyEndocrinology and Metabolism, 289(6): E960-E968.

Terada, S., \& Tabata, I. 2004. Effects of acute bouts of running and swimming exercise on PGC-1 $\alpha$ protein expression in rat epitrochlearis and soleus muscle. American Journal of Physiology-Endocrinology and Metabolism, 286(2): E208-E216.

Wu, Z., Puigserver, P., Andersson, U., Zhang, C., Adelmant, G., Mootha, V., \& Spiegelman, B. M. 1999. Mechanisms controlling mitochondrial biogenesis and respiration through the thermogenic coactivator PGC-1. Cell, 98(1): 115-124. 